\title{
Silent myocardial ischaemia in patients with proved coronary artery disease: a comparison of diabetic and non-diabetic patients
}

\author{
C J Ditchburn, J A Hall, M de Belder, A Davies, W Kelly, R Bilous
}

\begin{abstract}
Objective-To determine whether diabetic patients with coronary artery disease are more likely to experience silent myocardial ischaemia than subjects without diabetes.
\end{abstract}

Subjects-Patients undergoing coronary angiography at a regional cardiothoracic unit, identified as having diabetes from the local district diabetic register/ database.

Design-The coronary angiograms and exercise treadmill tests of 100 diabetic and 100 non-diabetic patients who all had significant coronary artery disease and maximal effort exercise tests were reviewed. Patients were individually matched for age group, gender, severity of coronary artery disease, and indication for treadmill test.

Outcome measures-Significant coronary artery disease was defined as a stenosis of greater than $50 \%$ diameter in at least one of the major coronary artery segments. Exercise tests were graded as positive if the electrocardiographic (ECG) recording showed planar or downsloping ST segment depression of $\geqslant 1 \mathrm{~mm}$ in more than two leads at $80 \mathrm{~ms}$ post J-point or if there was a blood pressure fall $\geqslant 10 \mathrm{~mm}$ $\mathrm{Hg}$ after an initial rise. A negative exercise test was defined as one in which the subject experienced no pain, had no ECG changes after maximal effort and had a normal blood pressure response. Subjects who did not experience any form of typical angina-type pain during a positive exercise test were defined as having an episode of silent ischaemia. Patients with symptomatic ischaemia were those who experienced typical angina-type pain with accompanying ST segment changes. Patients with a negative exercise test were defined as having "undetermined ischaemia". This category included all those without ECG evidence of myocardial ischaemia during the exercise test (with or without accompanying chest pain).

Results-In the diabetic patients, $34 \%$ had ECG evidence of silent ischaemia on treadmill testing compared with only $19 \%$ of the non-diabetic controls $(p<0.02)$.

Conclusions-This study shows that diabetic patients with proved coronary artery disease have a higher risk of developing silent myocardial ischaemia during exercise than non-diabetic patients.

(Postgrad Med f 2001;77:395-398)
Keywords: coronary heart disease; ischaemia, diabetes; prevalence

The majority of patients with ischaemic heart disease will experience myocardial ischaemia as anginal pain during exertion. However, the perceived severity of pain is not directly related to either the extent of coronary disease or the actual degree of ischaemia. ${ }^{1}$ This means that some patients with minor perfusion defects may experience severe anginal pain and some with severe disease will only have mild pain. Some patients will not experience pain at all (type I silent ischaemia) and as it is difficult to identify such patients the diagnosis is often made as an incidental finding during the investigation of another problem. The much more common form (type II) is little easier to diagnose because silent ischaemia in these patients is intermittent in nature.

To improve the identification of patients with type I silent ischaemia, it may be possible to focus on high risk subgroups, such as patients after myocardial infarction. Recent studies have also proposed that patients with diabetes mellitus are another subgroup, estimating the prevalence of silent ischaemia in diabetic patients to be $29 \%-69 \%$ compared with $5 \%-35 \%$ for non-diabetic patients. ${ }^{2-7}$

Although these studies are somewhat limited by their selection of patient groups or by the inclusion of small numbers of subjects $(n=<30)$, the fact remains that if diabetic patients do indeed experience more episodes of silent ischaemia than non-diabetic patients there are implications for screening and monitoring of all diabetic patients.

The aim of our study was to determine and compare the prevalence of silent ischaemia in two closely matched samples, one diabetic and one non-diabetic. A secondary objective was to find any possible associations between silent ischaemia and the type, duration, or complications of diabetes.

\section{Methods}

DIABETIC SUBJECTS

Diabetic patients $(n=783)$ of all ages who had been recorded (during the five year period 1992-97) as having either active or inactive ischaemic heart disease were initially located from a hospital database of registered diabetic patients. This PC based system running under Smartware II included all patients from the South Tees district of north east England who had been referred by their general practitioner to a consultant in diabetic medicine for further management of their diabetes. The names of these 783 patients were then cross referenced 
with a list of all patients who had undergone coronary angiography at the main district general hospital over the last five years $(\mathrm{n}=>6000)$ producing 131 subjects. An additional 107 diabetic subjects (total 238) were identified from a secretarial archive holding all coronary angiography lists for an eight month period of 1997. This was essentially a list of all patients ( $\mathrm{n}=>1500$ ) who had undergone cardiac catheterisation in one of two cardiac catheterisation laboratories. Known diabetic patients were identified as having either insulin dependent diabetes mellitus or non-insulin dependent diabetes on these lists so that the catheter laboratory nursing staff would be aware of patients susceptible to hypoglycaemia. This allowed the inclusion of diabetic patients who were undergoing the initial investigation of chest pain, and thus were not already recorded on the diabetic register as having established ischaemic heart disease. From both sources, only patients with significant coronary artery disease who had undergone maximum effort exercise treadmill testing within six months of angiography were selected. Any subjects taking digoxin or psychoactive drugs or who had aberrant conduction on the resting electrocardiogram (ECG) were excluded as these factors are known to confuse the interpretation of ST segment depression. Data were collected on gender, age at exercise test, indication for the test, and type of diabetes. Severity of coronary artery disease (one, two, three vessel or left main stem) was recorded from a computerised angiography database and details of exercise test performance were evaluated from the patient case notes.

NON-DIABETIC SUBJECTS

A list of all the remaining patients who had undergone angiography within the same five year period was then generated from the same angiography database $(n=>5000)$. Only patients who had significant coronary artery disease and who had undergone exercise test within six months of angiography were then selected from this list $(n=>1500)$. After applying identical exclusion criteria for the exercise tests and selecting only non-diabetic patients, a pool of 615 possible subjects was identified. To exactly match these controls to diabetic patients, a grid was constructed of diabetic males and females in five age bands of 10 year intervals with specific severities of coronary disease (table 1). One by one, patients were selected from the control pool at random by Microsoft Excel's built-in random number generator and were checked against the diabetic grid to see if they were appropriate matches. This meant that within each age band, individual diabetic patients could be matched to a control subject for gender, indication for exercise test, and severity of vessel disease. The selection process was repeated until all 100 controls had been found and identical data to that of the diabetic group were then collected for the control group by the same means.
INVESTIGATIONS

Angiography was performed in one of two cardiac catheterisation laboratories. The angiograms for every patient were recorded during the procedure on high resolution optical disc and later reviewed by a consultant cardiologist. The coronary anatomy data were recorded in a dedicated UNIX based Hewlett-Packard angiography database. Exercise testing was performed at the same hospital using a Marquette (MAX-1) treadmill system. All selected patients underwent the standard Bruce protocol and were referred for the investigation of either chest pain or established angina.

\section{DEFINITIONS}

Coronary artery disease was defined as being significant if there was a stenosis of greater than $50 \%$ diameter in at least one of the major coronary artery segments.

An exercise test was considered positive if the electrocardiographic recording showed planar or downsloping ST segment depression of $\geqslant 1 \mathrm{~mm}$ in more than two leads at $80 \mathrm{~ms}$ post J-point or if there was a blood pressure fall $\geqslant 10$ $\mathrm{mm} \mathrm{Hg}$ after an initial rise. A negative exercise test was defined as one in which the subject experienced no pain, had no ECG changes after maximal effort and had a normal blood pressure response.

Maximal effort was defined as the attainment of at least $80 \%$ of the maximum predicted heart rate (220 minus age) with a normal blood pressure response. An equivocal exercise test was defined as one not meeting either positive or negative criteria.

Patients with a positive exercise test were subdivided into two mutually exclusive categories to determine the presence of either silent or symptomatic ischaemia. Subjects meeting positive exercise test criteria who did not experience any form of simultaneous typical anginatype pain (discomfort in the chest, jaw, or arm) were defined as having an episode of silent ischaemia. Patients with symptomatic ischaemia were those who experienced typical angina-type pain with accompanying ST segment changes.

Patients with a negative exercise test were defined as having "undetermined ischaemia". This category included all those without electrocardiographic evidence of myocardial

Table 1 Number of diabetic patients with various severities of coronary artery disease grouped into age ranges

\begin{tabular}{lllll}
\hline Age range (years) & $1 V D$ & $2 V D$ & $3 V D$ & $L M C A$ \\
\hline Males & & & & \\
$30-39$ & 1 & 1 & 0 & 0 \\
$40-49$ & 2 & 3 & 5 & 0 \\
$50-59$ & 3 & 7 & 17 & 4 \\
$60-69$ & 4 & 9 & 9 & 3 \\
$70-79$ & 1 & 0 & 6 & 0 \\
Females & & & & \\
$30-39$ & 1 & 1 & 0 & 0 \\
$40-49$ & 1 & 0 & 1 & 0 \\
$50-59$ & 4 & 3 & 6 & 0 \\
$60-69$ & 0 & 2 & 5 & 0 \\
$70-79$ & 0 & 0 & 1 & 0
\end{tabular}

$1 \mathrm{VD}=1$ vessel disease; $2 \mathrm{VD}=2$ vessel disease $3 \mathrm{VD}=3$ vesse disease; $\mathrm{LMCA}=$ disease in the left main coronary artery. 
Table 2 Number of patients in both diabetic and non-diabetic groups having either negative exercise tests or positive exercise tests with ischaemia

\begin{tabular}{llll}
\hline & $\begin{array}{l}\text { Positive exercise test with } \\
\text { symptomatic ischaemia }\end{array}$ & $\begin{array}{l}\text { Positive exercise test with } \\
\text { silent ischaemia }\end{array}$ & $\begin{array}{l}\text { Negative } \\
\text { exercise test }\end{array}$ \\
\hline Diabetics $(\mathrm{n}=100)$ & 32 & 34 & 34 \\
Non-diabetics $(\mathrm{n}=100)$ & 45 & 19 & 36 \\
\hline
\end{tabular}

ischaemia during the exercise test (with or without accompanying chest pain).

ANALYSIS

The data collected from both groups was tabulated using Microsoft Excel 7.0 and analysed using the in-built statistical functions. The resulting prevalences were compared and analysed in $2 \times 2$ contingency tables for statistical significance using the $\chi^{2}$ test.

\section{Results}

DIABETIC GROUP

Of the total 238 diabetic patients undergoing angiography, 186 had significant coronary artery disease and 144 of these patients had undergone Bruce protocol exercise treadmill testing within six months of angiography. After applying the remaining exclusion criteria, and after excluding inadequate or equivocal tests a total of 104 diabetic patients remained who had either positive or negative exercise tests. From these, 100 were randomly selected by Microsoft Excel's built-in random number function and consisted of 75 males (mean age 58.9 years, $S D=8.6$ ) and 25 females (mean age 57.1 years, $S D=10.2)$. Sixty six of the diabetic patients had positive exercise tests and silent ischaemia was detected in 34 patients $(95 \%$ confidence interval (CI) 24.7 to 43.3). Symptomatic ischaemia was detected in the other 32 diabetic patients with positive exercise tests. This indicates that approximately half of all the diabetic patients with positive exercise tests had silent ischaemia. Of the 82 patients with type II diabetes, 30 (36\%) had silent ischaemia, compared with only four of the 18 patients $(22 \%)$ with type I diabetes $(0.1<\mathrm{p}<0.5)$.

NON-DIABETIC GROUP

By writing a computer program to search the catheter laboratory database, it was possible to identify 3586 non-diabetic patients with significant coronary artery disease. Only 893 $(25 \%)$ of these patients had undergone conclusively positive or negative maximal exercise treadmill testing within six months of angiography, and only 615 met all the inclusion/ exclusion criteria. After selecting 100 matches from the control pool, 457 had been rejected as unsuitable matches, 45 were duplicate matches, and 113 patients were left in the control pool who had not been picked for matching. The final control group consisted of 75 males (mean age 58.2 years, SD 8.0) and 25 females (mean age 56.4 years, SD 8.5). Of the 64 non-diabetic patients with positive exercise tests, silent ischaemia was detected in three females and 16 males, a total of 19 patients ( $95 \% \mathrm{CI}=11.3$ to 26.7 ). Symptomatic ischaemia was detected in the other 45 patients. The remainder of the non-diabetic patients had negative exercise tests.
COMPARISON

Overall, the prevalence of silent ischaemia between the two groups differs quite markedly at $34 \%$ and $19 \%$ (table 2). The difference of $15 \%$, has a standard error of $6.15 \%$ and a ratio of difference to standard error of 2.43 $(p<0.046)$. This statistical significance is confirmed by $\chi^{2}$ testing, which gives a value for $\chi^{2}$ of $5.78(\mathrm{p}<0.02,1 \mathrm{df})$.

\section{Discussion}

This study of closely matched diabetic and non-diabetic patients found that when diabetic patients with established coronary artery disease undergo maximal effort treadmill testing, significantly more of them will display evidence of myocardial ischaemia without feeling angina-type pains than their matched nondiabetic counterparts. The study was specifically designed to test the hypothesis that diabetes confers a higher risk of silent ischaemia by taking a homogeneous population of patients with angiographically proved coronary artery disease and retrospectively reviewing their exercise treadmill test data for any evidence of silent ischaemia. Starting with this overall population, two samples were drawn and matched for age, gender, and indication for the treadmill test, so that the only major variable between both samples was a confirmed diagnosis of diabetes mellitus. By having two reasonably large samples, the resulting data do indeed add support to the hypothesis under test.

This study has a number of limitations. First, the patient samples could have been matched more closely by either selecting patients off all antianginal therapy at the time of the treadmill test or by matching individual patients with identical medication. Secondly, the patients in the non-diabetic sample did not undergo fasting blood glucose testing to exclude possible undiagnosed diabetes. Ideally, a prospective study should be undertaken including these extra refinements, taking very large samples from a wider demographic area.

Silent ischaemia remains a problem in both diabetic and non-diabetic populations. No therapeutic regimen has yet been shown to improve the prognosis of patients with silent ischaemia, although one study identified a high risk subgroup of patients with silent ischaemia after myocardial infarction and suggested they undergo early therapeutic revascularisation to improve long term mortality. ${ }^{8}$ Another study suggested the use of low dose aspirin to reduce mortality in patients with silent ischaemia after either a non-Q wave infarct or an episode of unstable angina. ${ }^{9}$

Because diabetics appear to have a higher prevalence of silent myocardial ischaemia, it has been suggested that they may undergo routine screening with an annual exercise test. However, Sayer and Timmis have suggested that such screening cannot be recommended to all diabetic patients because of the low diagnostic yield from non-invasive testing. ${ }^{10}$ Rutter et al do however report that only asymptomatic insulin dependent patients with microalbuminuria may benefit from screening and 
appropriate intervention. ${ }^{11}$ Our study suggests that patients with chest pain and angina should undergo exercise testing, and patients with probable angina equivalents (for example, exertional breathlessness) should also be evaluated.

\section{Conclusion}

Diabetics with coronary artery disease have a higher prevalence of silent myocardial ischaemia than matched non-diabetic patients with coronary artery disease. The development of silent ischaemia does not appear to be related to the type, duration, or complications of diabetes.

1 Quyyumi AA, Wright CM, Mockus LJ, et al. How important is a history of chest pain in determining the degree of ischaemia in patients with angina pectoris? $\mathrm{Br}$ Heart $\mathcal{f}$ 1985;54:22-6.

2 Koistinen MJ. Prevalence of asymptomatic myocardial ischaemia in diabetic subjects. BMF 1990;301:92-5.
3 Murray DP, O'Brien T, Mulrooney R, et al. Autonomic dysfunction and silent myocardial ischaemia on exercise testing function and silent myocardial ischaemia on exerci

4 Ahluwalia G, Jain P, Chugh SK, et al. Silent myocardial ischaemia in diabetics with normal autonomic function. Int 7 Cardiol 1995;48:147-53.

5 Parmley WW. Prevalence and clinical significance of silent myocardial ischaemia. Circulation 1989;80(6 suppl IV): 68-73.

6 Sharma SN, Bajaj R, Kaul U, et al. Silent myocardial ischaemia in patients with angiographically proven coronary artery disease. Indian Heart 7 1989;41:158-61.

7 Mulcahy D, Keegan J, Crean P, et al. Silent myocardial ischaemia in chronic stable angina: a study of its frequency and characteristics in 150 patients. Br Heart $\mathcal{F} 1988 ; 60: 417-$ 23.

8 de Belder M, Skehan D, Pumphrey C, et al. Identification of a high risk subgroup of patients with silent ischaemia after myocardial infarction: a group for early therapeutic revascumyocardial infarction: a group for early

9 Nyman I, Larsson H, Wallentin L. Prevention of serious cardiac events by low-dose aspirin in patients with silent myocardial ischaemia. The Research Group on Instability in Coronary Artery Disease in Southeast Sweden. Lancet 1992;340:497-501.

10 Sayer JW, Timmis AD. Investigation of coronary artery disease in diabetics: is screening of asymptomatic patients necessary? Heart 1997;78:525-6.

11 Rutter MK, Marshall SM, McComb JM. Coronary artery disease and diabetes. Heart 1997;78:527-8. 